\title{
1 Suppression of sound radiation to far field of near-field acoustic 2 communication system using evanescent sound field
}

\author{
3 Ayaka Fujii ${ }^{1}$, Naoto Wakatsuki ${ }^{2 *}$, and Koichi Mizutani ${ }^{2}$ \\ $4 \quad{ }^{1}$ Graduate School of Systems and Information Engineering, University of Tsukuba, Tsukuba, Ibaraki \\ 5 305-8573, Japan \\ $6{ }^{2}$ Faculty of Engineering, Information and Systems, University of Tsukuba, Tsukuba, Ibaraki 305-8573, \\ 7 Japan
}

8

A method of suppressing sound radiation to the far field of a near-field acoustic communication system using an evanescent sound field is proposed. The amplitude of the evanescent sound field generated from an infinite vibrating plate attenuates exponentially with increasing a distance from the surface of the vibrating plate. However, a discontinuity of the sound field exists at the edge of the finite vibrating plate in practice, which broadens the wavenumber spectrum. A sound wave radiates over the evanescent sound field because of broadening of the wavenumber spectrum. Therefore, we calculated the optimum distribution of the particle velocity on the vibrating plate to reduce the broadening of the wavenumber spectrum. We focused on a window function that is utilized in the field of signal analysis for reducing the broadening of the frequency spectrum. The optimization calculation is necessary for the design of window function suitable for suppressing sound radiation and securing a spatial area for data communication. In addition, a wide frequency bandwidth is required to increase the data transmission speed. Therefore, we investigated a suitable method for calculating the sound pressure level at the far field to confirm the variation of the distribution of sound pressure level determined on the basis of the window shape and frequency. The distribution of the sound pressure level at a finite distance was in good agreement with that obtained at an infinite far field under the condition generating the evanescent sound field. Consequently, the window function was optimized by the method used to calculate the distribution of the sound pressure level at an infinite far field using the wavenumber spectrum on the vibrating plate. According to the result of comparing the distributions of the 
sound pressure level in the cases with and without the window function, it was confirmed that the area whose sound pressure level was reduced from the maximum level to $-50 \mathrm{~dB}$ was extended. Additionally, we designed a sound insulator so as to realize a similar distribution of the particle velocity to that obtained using the optimized window function. Sound radiation was suppressed using a sound insulator put above the vibrating surface in the simulation using the three-dimensional finite element method. On the basis of this finding, it was suggested that near-field acoustic communication which suppressed sound radiation can be realized by applying the optimized window function to the particle velocity field.

\section{Introduction}

The near-field wireless communication systems included in various devices such as mobile phones can easily perform one-on-one communication. ${ }^{1)}$ Such communication systems are also utilized for electronic payments and require a high security level because data communication can be performed only when a device approaches sufficiently close to an intended device. ${ }^{2)}$ Some methods using IC cards that are in widespread use have a common standard and require corresponding hardware. ${ }^{3)}$ On the other hand, we have studied an acoustic transducer for a near-field acoustic communication system using an evanescent sound field generated near the surface of a vibrating plate, as shown in Fig. 1. ${ }^{4)}$ Extra hardware for data communication is unnecessary because the near-field acoustic communication system requires only a loudspeaker and a microphone, which are already installed in mobile devices.

The proposed acoustic transducer consists simply of a single vibrating plate, which

\footnotetext{
*E-mail: wakatuki@iit.tsukuba.ac.jp
} 
1 is made of acrylic with a thickness of $2 \mathrm{~mm}$, and two actuators. Figure 2 shows the

2 frequency characteristics of the wavenumber $k_{\mathrm{p}}$ of a bending wave propagating on an

3 infinite acrylic plate with a thickness of $2 \mathrm{~mm}$. The frequency of the bending wave

4 is expressed as $f$ hereafter. The density of the plate is $1,225 \mathrm{~kg} / \mathrm{m}^{3}$ and its Young's

5 modulus is $5.61 \mathrm{GPa}$. In addition, the dashed line shows the frequency characteristics

6 of the wavenumber $k$ of a plane wave in air when the sound speed in air is $340 \mathrm{~m} / \mathrm{s}$.

7 An evanescent sound field is generated near the surface of the vibrating plate when $f$ is

8 under $15 \mathrm{kHz}$ because the wavenumber $k_{\mathrm{p}}$ of the bending wave on the vibrating plate is

9 larger than the wavenumber $k$ of the plane wave in air. ${ }^{5)}$ The amplitude of the evanescent

10 sound field generated from an infinite vibrating plate attenuates exponentially with

11 increasing distance from the surface of the vibrating plate. ${ }^{6-8)}$ Therefore, it is possible

12 to perform data communication without leaking acoustic data. Additionally, the existing

13

acoustic communication system for mobile devices transmits data at a low speed because the frequency band is limited to near the maximum auditory frequency of $18 \mathrm{kHz}$ to prevent the radiation of sound that may be unpleasant for people. ${ }^{9,10)}$ In contrast, the proposed acoustic transducer can increase the data transmission speed using a wide frequency band including auditory frequencies because the generated sound does not reach the audible spatial area in theory. However, a discontinuity of the sound field exists at the edge of the finite vibrating plate in practice. This broadens the wavenumber spectrum, and the vibration plate fails to satisfy the condition of $k_{\mathrm{p}}>k$.

1 In consequence, a sound wave radiates over the evanescent sound field, causing the 22 leakage of acoustic data and sound noise. Therefore, we need a method for reducing the 3 broadening of the wavenumber spectrum or a vibrating plate that always satisfies the 
1 condition of $k_{\mathrm{p}}>k$. Since the proposed acoustic transducer consists of a homogeneous

2 vibrating plate, $k_{\mathrm{p}}$ can be increased by reducing the thickness or by choosing a material

3 with a high density and low Young's modulus. ${ }^{11)}$ However, the area in which data

4 communication can be performed becomes extremely narrow because the attenuation

5 of the sound pressure with increasing distance from the surface of the vibrating plate

6 becomes very large with increasing $k_{\mathrm{p}}$. Accordingly, we must consider the use of near-

7 field acoustic communication.

8 In this study, we propose a method for suppressing sound radiation by reducing the

9 broadening of the wavenumber spectrum. First, the optimal distribution of the particle

10 velocity on the vibrating plate is calculated so as to make the sound pressure level

11 small at the boundary of the spatial area in which data communication is forbidden.

12 We focus on a window function that is utilized in the field of signal analysis to reduce the broadening of the frequency spectrum. The window function is optimized by vary-

14 ing its length and its type of shoulder. To confirm the possibility of suppressing sound radiation, we calculate the distribution of the sound pressure level when the optimized window function is applied to the particle velocity field. Next, we design a sound inusing the optimized window function. We investigate the suppression of sound radiation upon placing a sound insulator above the vibrating surface by simulation using the three-dimensional finite element method (FEM). 


\section{2. Wavenumber spectrum of wave field on vibrating plate and window functions}

2 Figure 3 shows sound generation from a bending wave propagating on a finite plate.

3 An evanescent sound field is generated near the surface of the vibrating plate when the

4 wavenumber of the bending wave on the vibrating plate $k_{\mathrm{p}}$ is larger than that of the

5 plane wave in air $k$. On the other hand, when $k_{\mathrm{p}}$ is smaller than $k$, the sound is radiated

6 similarly to a beam with a uniform level and a constant angle, as shown in Fig. 3(a).

7 Figure 4 shows the wavenumber spectrum of a bending wave propagating on an infinite

8 vibrating plate with the wavenumber $k_{\mathrm{p}}$ and that obtained by applying a finite rectangu-

9 lar window to the particle velocity field. The solid line and dashed line show the results

10 for the infinite vibrating plate and finite rectangular window, respectively. In the case

11

12

13

of the infinite vibrating plate, only the component with the wavenumber $k_{\mathrm{p}}$ exists in the particle velocity field, and no extra components of the wavenumber spectrum, called spurious signals, appear. Thus, the evanescent wave dominates the sound field. On the other hand, in the case of the finite rectangular window, the wavenumber spectrum has a main lobe with a finite bandwidth and the spurious signal is large. Additionally, a nonevanescent sound wave radiates since a large power exists at wavenumbers smaller than $k$, the wavenumber of a plane wave in air. Therefore, the window function is necessary for suppressing sound radiation and securing a spatial area for data communication.

Here, we discuss the window functions used in this study and their characteristics.

We use a triangular window, a Hann window, a cosine window, and a Blackman window.

21 Figures 5 and 6 respectively show the window functions and their wavenumber spectra.

2 The solid line, dashed line, chain line, and two-dot chain line in Fig. 5 show the trian- 
1 gular window, Hann window, cosine window, and Blackman window, respectively. It is

2 confirmed that the triangular window and cosine window have a large side lobe level.

3 However, the main lobe of the cosine window is the narrowest. The width of the main

4 lobe of the Hann window is identical to that of the triangular window. Nevertheless,

5 the spurious level of the Hann window is lower than that of the triangular window. On

6 the other hand, the Blackman window has the smallest spurious level and the widest

7 main lobe. Since these windows have different characteristics, optimization calculation

8 is necessary to determine the window function most suitable for data communication.

9 Figure 7 shows an example of a window function with a shoulder part consisting of a

10 cosine roll-off. This window is called the cosine roll-off window. The window function

11 is optimized by varying the ratio of the shoulder part to the flat part of the window,

12 as shown in Fig. 7. To secure a large sound pressure level in the receivable area, the

13 minimum value of the window inside the receivable area is unity.

14 In addition, it is predicted that the sound pressure level of a sound wave leaking to a far field changes with the ratio of $k_{\mathrm{p}}$ to $k$. We have to take into account the

16 fact that the wavenumber $k_{\mathrm{p}}$ is defined as a function of the frequency in the case of

17 the vibrating plate, as shown in Fig. 2. Therefore, we calculate the variation of the sound pressure level at the far field with respect to the frequency and window shape. are investigated in Sect. 3.1. 


\section{3 . Sound pressure level with respect to frequency and window shape}

\subsection{Calculation of sound pressure level at far field of vibrating plate}

3 In order to evaluate the sound wave leaking to the far field with respect to the frequency

4 and window shape under the condition that an evanescent sound field is generated,

5 we investigated calculation methods for the sound pressure level at a far field from a

6 vibrating plate. First, we used a calculation method for the sound pressure level at a

7 finite distance from the vibrating plate as a realistic method. Acoustic holography is a

8 well-known method for calculating the sound field on a plane using another sound field

9 on a parallel plane. ${ }^{12-15)}$ Acoustic holography can be utilized to evaluate the difference in

10

sound pressure level between the surface of the vibrating plate and an adjacent parallel surface at a distance from the vibrating plate. ${ }^{16-20)}$ However, we should consider a sound wave radiating at an angle given by following equation when the wavenumber $k_{\mathrm{p}}$ of the bending wave is smaller than the wavenumber $k$ of the plane wave in air.

$$
\tan \theta=\sqrt{k^{2} / k_{\mathrm{p}}^{2}-1}
$$

The surface at the far field used for evaluating the sound radiation in a finite length is not on a parallel plane but on a spherical surface. Therefore, we used the Rayleigh integral to calculate the sound pressure at any observation point. ${ }^{21-23)}$ Figure 8 shows the position of the vibrating plate of the proposed acoustic transducer on the $x y$-plane and the calculation area of the sound pressure level at the far field. The length $L$ of the vibrating plate was $200 \mathrm{~mm}$. The calculation area, in which data communication is forbidden, was defined as the outside of a semicircle of $500 \mathrm{~mm}$ radius that was sufficiently far from the center of the vibrating plate. The boundary of the calculation area is called 
1 the evaluation boundary. The calculation interval at the evaluation boundary was one-

2 tenth of the maximum wavelength $\lambda$ of the radiated sound. It was assumed that the

3 proposed acoustic transducer, which was surrounded by an infinite baffle at $z=0$,

4 vibrated with a bending wave at the particle velocity $v(x, t)$. Additionally, the particle

5 velocity distribution in the $y$-direction was assumed to be uniform within the width of

6 the vibrating plate. When the sound pressure radiating from a point $\boldsymbol{r}_{\mathbf{0}}=\left(x_{0}, y_{0}, z_{0}\right)$

7 on the vibrating plate is observed at a point $\boldsymbol{r}=(x, y, z)$, the instantaneous velocity

8 potential $w(\boldsymbol{r}, t)$ at time $t$ is given by:

$$
\begin{aligned}
w(\boldsymbol{r}, t) & =\iint_{S} \frac{1}{2 \pi\left|\boldsymbol{r}-\boldsymbol{r}_{\mathbf{0}}\right|} v\left(t-\frac{\left|\boldsymbol{r}-\boldsymbol{r}_{\mathbf{0}}\right|}{c}\right) d x_{0} d y_{0}, \\
v\left(t-\frac{\left|\boldsymbol{r}-\boldsymbol{r}_{\mathbf{0}}\right|}{c}\right) & =u_{\mathrm{p}} \exp \left(-j k_{\mathrm{p}} x\right) \exp \left[j \omega\left(t-\frac{\left|\boldsymbol{r}-\boldsymbol{r}_{\mathbf{0}}\right|}{c}\right)\right],
\end{aligned}
$$

9 where $S, c$, and $u_{\mathrm{p}}$ are the vibrating surface, the sound speed of the plane wave in air, and the amplitude of the bending vibration, respectively. The following equation was utilized to perform harmonic analysis when $\exp (j \omega t)$ is separated from Eq. (3) in this study:

$$
w(\boldsymbol{r})=\iint_{S} \frac{1}{2 \pi\left|\boldsymbol{r}-\boldsymbol{r}_{\mathbf{0}}\right|} u_{\mathrm{p}} \exp \left(-j k_{\mathrm{p}} x\right) \exp \left(-j \omega \frac{\left|\boldsymbol{r}-\boldsymbol{r}_{\mathbf{0}}\right|}{c}\right) d x_{0} d y_{0}
$$

The calculation intervals in the $x$ - and $y$-directions on the vibrating surface were one14 hundredth of the maximum wavelength $\lambda_{\mathrm{p}}$ and one-tenth of $\lambda_{\mathrm{p}}$ for the bending wave.

We also investigated a calculation method for the sound pressure level at an infinite distance. The method employs the Frounhofer approximation to define the wavenumber spectrum of a vibrating surface as the sound pressure level at an infinite far field. ${ }^{24)}$ The wavenumber spectrum was calculated by performing a Fourier transform with respect to the distribution of the sound pressure on the vibrating plate. The sound pressure 
1 level at the angle of an infinite distance was calculated by converting the wavenumber

$2 \quad k_{\mathrm{p}}$ into the angle using Eq. (1). The region of the vibrating plate and the calculation

3 area on the $x$-axis were $-100 \leq x \leq 100 \mathrm{~mm}$ and $-1,000 \leq x \leq 1,000 \mathrm{~mm}$, respectively. The

4 calculation interval was $\lambda_{\mathrm{p}} / 100$. For both methods, we compared the sound pressure

5 level at the far field under the condition where an evanescent sound field is generated,

6 i.e., $k_{\mathrm{p}}>k$, and that under the condition radiating sound wave, i.e., $k_{\mathrm{p}}<k$. Therefore,

7 a bending wave propagating at frequencies of 6 and $30 \mathrm{kHz}$ on the acrylic plate shown

8 in Fig. 2 was assumed.

\subsection{Results}

We compared the distributions of the sound pressure level at a finite distance and an infinite distance from the vibrating plate. The $z$-axis was set to 0 rad. The sound pressure level was normalized using the maximum value in the range from $-\pi / 2$ to $\pi / 2$. Figures 9(a) and 9(b) show the angular characteristics of the sound pressure level at frequencies of 6 and $30 \mathrm{kHz}$, respectively. The solid line and dotted line show the sound pressure level at the evaluation boundary and that obtained at an infinite distance.

In the case of $f=6 \mathrm{kHz}$ under the condition that an evanescent sound field is generated, the angular characteristics of the sound pressure level at the finite distance were in good agreement with those at the infinite distance, as shown in Fig. 9(a). In addition, the differences in sound pressure level with respect to the angle were small. On the other hand, in the case of $f=30 \mathrm{kHz}$ under the condition that a sound wave is radiated, the sound pressure level at the infinite distance had a maximum value with a sharp peak at $\pi / 4$. The difference in sound pressure level between the maximum value 
1 of the main lobe and that of the adjacent peak was approximately $13 \mathrm{~dB}$. The sound

2 pressure level at the evaluation boundary also had a maximum value at $\pi / 4$; however,

3 the main lobe was wider than that for the infinite distance. It is inferred that the sound

4 wave is radiated similarly to a beam from the finite vibrating plate, and the beam width

5 was too long relative to the circumference of the semicircle of the evaluation boundary,

6 as shown in Fig. 3(a). Consequently, the sound pressure level at the evaluation boundary

7 was in disagreement with that obtained at the infinite distance.

\section{$8 \quad 3.3$ Discussion}

9 We aim to design a window function that reduces the maximum sound pressure level at a far field to suppress the leakage of sound waves. Therefore, the error at the dip shown in

Fig. 8 is less important when the peak values for both methods are identical. According to Fig. 9(a), differences in the level and the position at the peak between the cases of a finite distance and an infinite distance are not observed under the condition that an evanescent sound field is generated. Additionally, the method using the wavenumber spectrum on the vibrating plate allows easier calculation than that using Eq. (4). Since this study aims to suppress the radiation component of the evanescent sound field, we evaluate the sound pressure level at the far field using the method in which the wavenumber spectrum on the vibrating plate is calculated.

\section{Optimization of window function}

\subsection{Frequency characteristics of sound pressure level with respect to window shape}

21 The distribution of the particle velocity should be designed to suppress the sound pressure level at the far field. Therefore, the sound pressure level at the far field from 
1 the vibrating plate with respect to the frequency and window shape is calculated. Here,

2 we set some limiting conditions for the window function that determine the distribution

3 of the particle velocity on the vibrating surface. First, the entire length of the window

4 function was identical to the length of the vibrating plate of the proposed acoustic

5 transducer, i.e., $L=200 \mathrm{~mm}$. The window function was symmetrical about the center

6 of the vibrating plate. Next, the ratio of the shoulder part to the flat part of the window

7 was changed by varying the shoulder length $l$ from 0 to $100 \mathrm{~mm}$, as shown in Fig. 7 .

8 In addition, it was inferred that the sound pressure level in the receivable area above

9 the vibrating plate becomes small upon increasing the ratio of the shoulder part to

10 the flat part. Therefore, the area that the data should reach was defined as the range

$11-50 \leq x \leq 50 \mathrm{~mm}$, and the minimum value of the window inside the area was set as 1 .

12 Additionally, the window functions whose shoulders consisted of the triangular window,

13

14

15

16

17

Hann window, cosine window, and Blackman window are called triangular-type window, cosine roll-off window, cosine-type window, and Blackman-type window, respectively. The maximum sound pressure level within the range under the condition for which sound is radiated, $k_{\mathrm{p}}<k$, was obtained by calculating the wavenumber spectrum on the vibrating plate when the window functions were applied to the particle velocity field. Finally, we must consider the fact that the proposed acoustic transducer was designed for data communication by mobile devices. Therefore, the frequency characteristics of the sound pressure level were calculated in the frequency band $1 \leq f \leq 15 \mathrm{kHz}$, i.e., under the theoretical condition that an evanescent sound field is generated that can be received by the microphone in a mobile device. The frequency interval in the calculation was $0.1 \mathrm{kHz}$. 
1

4 Blackman-type window, respectively. In addition, it is clear from Figs. 10(a) - 10(d)

5 that the sound pressure level abruptly increased at approximately $f=10 \mathrm{kHz}$. The

6 frequency band below $15 \mathrm{kHz}$ theoretically satisfies the condition that an evanescent

7 sound field is generated when the vibrating surface has an infinite length. However, it

8 was inferred that the main lobe of the wavenumber spectrum increased in width owing

Figure 10 shows the sound pressure level at the far field from the vibrating plate with respect to the frequency for different window shapes. Figures $10(a)-10(d)$ show the results for the triangular-type window, cosine roll-off window, cosine-type window, and to the length being finitely cut off in all windows. Therefore, part of the main lobe was already smaller than the wavenumber $k$ of the plane wave in air at $f=10 \mathrm{kHz}$. The fact that the sound pressure level of the radiated sound is high in all windows indicates that the frequency band above $f=10 \mathrm{kHz}$ is unsuitable for limiting the communication area to near the proposed acoustic transducer. In addition, the maximum sound pressure level at the far field changed with the frequency, the length of the shoulder, and the type of window. The window function in which the maximum sound pressure level is small has to be designed to suppress sound radiation. However, the proposed acoustic transducer aims to transmit acoustic data with a wide frequency bandwidth to allow high data transmission speeds. It is also necessary to prevent sound from radiating at a specific frequency so that sound noise as well as acoustic data do not leak. Therefore, 20 we optimize the window function in the frequency band $1 \leq f \leq 10 \mathrm{kHz}$ so that the maximum sound pressure level at the far field is minimized. 
1

2 The limiting conditions in the optimization of the window function follow those in the 3 previous section, as shown in Fig. 7. Here, we describe the evaluation function use in the

4 optimization. Similarly, the sound pressure level was calculated using the wavenumber 5 spectrum on the vibrating surface by the same method as that in the previous section.

6 The maximum sound pressure level of the radiated sound at the infinite far field from 7 the vibrating plate was calculated. The window function in which the maximum sound

\subsection{Procedure of optimization of window function}

pressure level at the far field has the smallest value in the frequency band $1 \leq f \leq 10 \mathrm{kHz}$

was obtained. Under these conditions, the optimization involves the following steps.

(1) The maximum sound pressure level $L_{\max }$ at an infinite far field in the frequency band $1 \leq f \leq 10 \mathrm{kHz}$ for each shoulder length in the range of $0 \leq l \leq 100 \mathrm{~mm}$ is calculated.

(2) The shoulder length of the optimal window that gives the minimum value of $L_{\max }$ in step (1) is searched for.

Using the evaluation function, the optimized window that suppresses sound radiation at the far field when using the proposed acoustic transducer for data communication was determined.

\subsection{Results}

Figure 11 shows $L_{\max }$ with respect to the shoulder length of the window in the frequency band $1 \leq f \leq 10 \mathrm{kHz}$. The solid line, dashed line, chain line, and two-dot chain line show $L_{\max }$ for the triangular-type window, cosine roll-off window, cosine-type window, and Blackman-type window, respectively. The shoulder lengths giving the minimum value 
1 of $L_{\max }$ for the triangular-type window, cosine roll-off window, cosine-type window, and

2 Blackman-type window were 80, 84, 100, and 74 mm, respectively, as shown in Fig. 11.

3 The corresponding values of $L_{\max }$ were $12.90,18.98,19.35$, and $21.51 \mathrm{~dB}$, respectively.

4 Thus, the triangular-type window with $l=80 \mathrm{~mm}$, for which the minimum value of

$5 L_{\max }$ was obtained among the four types of windows, was determined as the optimized

6 window.

$7 \quad$ The distribution of the sound pressure level on the $x z$-plane $(y=0)$ was calculated 8 using the Rayleigh integral to confirm that the sound pressure level depends on the 9 presence or absence of the optimized window. The calculation area in the sound field was $-500 \leq x \leq 500 \mathrm{~mm}$ and $0 \leq z \leq 1,000 \mathrm{~mm}$. The calculation interval was $1 \mathrm{~mm}$. Figure 12 shows the distribution of the sound pressure level on the $x z$-plane $(y=0)$. Figures 12(a) and 12(b) show the distributions of the sound pressure level without and with the optimized window when $f=6 \mathrm{kHz}$, respectively. The maximum sound pressure level in each sound field was normalized as $0 \mathrm{~dB}$, and the level is shown down to -50 $\mathrm{dB}$.

First, we focused on the area whose level was in the range from 0 to $-10 \mathrm{~dB}$. According to Figs. 12(a) and 12(b), this area was included within the area $-50 \leq x \leq 50$ mm. This suggests that a sufficiently high sound pressure level can be secured within the area defined as the minimal spatial area for data communication. Next, we focused on the sound pressure level at the far field. In the case without the optimized window, the area where the sound pressure level decreased from -40 to $-50 \mathrm{~dB}$ existed at $z=$ 1,000 mm, as shown in Fig. 12(a). On the other hand, in the case with the optimized window, the area where sound pressure level decreased from -40 to -50 dB existed within 
$1-150 \leq x \leq 500 \mathrm{~mm}$ and $0 \leq z \leq 250 \mathrm{~mm}$, as shown in Fig. 12(b).

\subsection{Discussion}

3 It was predicted that the Blackman window is most effective for the suppression of sound

4 radiation in the case of a single frequency since the spurious wavenumber spectrum 5 is the smallest. However, an acoustic transducer using an evanescent sound field was 6 proposed for near-field acoustic communication with the acoustic data transmitted with 7 a frequency bandwidth. Therefore, we had to secure a large sound pressure level in the 8 receivable area as well as suppress the sound wave leakage. The results indicated that 9 a large sound pressure level can be secured within the area defined as the minimal spatial area for data communication. Furthermore, the sound pressure level decreased in a shorter distance than in the case without the optimized window. Therefore, it is possible to design an optimized function using the evaluation function and to limit the area that transmits acoustic data using the optimal window while securing a high sound pressure level near the vibrating plate. According to these results, near-field acoustic communication with suppressed sound radiation can be achieved by applying the optimal window to the particle velocity field.

\section{FEM simulation on effect of covering sound source with porous material to} suppress sound radiation

\subsection{Simulation procedure using three-dimensional finite element method}

To confirm the feasibility of distributing the particle velocity field in reality, we simulate the particle velocity above the vibrating surface when a sound insulator made of a porous material is placed in the evanescent sound field. Porous materials are often used 
1 for sound absorption and insulation. The characteristic impedance $Z$ and propagation 2 constant $\gamma$ of a porous material are expressed as the following functions of the flow 3 resistivity $R_{\mathrm{f}}$ and frequency $f$ in the Miki model: ${ }^{25,26)}$

$$
\begin{aligned}
Z(f) & =R(f)+j X(f), \\
\gamma(f) & =\alpha(f)+j \beta(f), \\
R(f) & =\rho c\left[1+0.070\left(\frac{f}{R_{\mathrm{f}}}\right)^{-0.632}\right], \\
X(f) & =-0.107 \rho c\left(\frac{f}{R_{\mathrm{f}}}\right)^{-0.632}, \\
\alpha(f) & =0.160 \frac{\omega}{c}\left(\frac{f}{R_{\mathrm{f}}}\right)^{-0.618}, \\
\beta(f) & =\frac{\omega}{c}\left[1+0.109\left(\frac{f}{R_{\mathrm{f}}}\right)^{-0.618}\right],
\end{aligned}
$$

4 where $c$ and $\omega$ are the sound velocity in air and the angular frequency, respectively.

5 Here, the distribution of the particle velocity in the case that a porous material, as an

6 example of a sound insulator, is placed above the vibrating surface was calculated by

7 FEM simulation. The evanescent sound field was generated by interchanging the normal

8 particle velocity of a bending wave traveling in the positive $x$-direction on the vibrating 9 surface and that of the sound field. The pressure of the sound field was input into the 10 vibrating surface. The bending wave was assumed to be propagating with a frequency 11 of $f=6 \mathrm{kHz}$ on the acrylic plate in accordance with Fig. 2. The shape of the sound 12 insulator should be designed so that the distribution of the particle velocity above 13 the sound insulator is extremely close to that obtained using the optimized window 14 function. However, the attenuation characteristics in the porous material have not been analyzed in detail when the material is placed in the evanescent sound field. On the 
1 other hand, it is well known that the amplitude decreases with increasing thickness of

2 the porous material in the case of a plane wave. Therefore, we investigate the possibility

3 of distributing the particle velocity above the vibrating surface using a sound insulator

4 with a continuous thickness distribution. The sound insulator had a wedge shape so

5 that the distribution of the particle velocity at the edge of the vibrating surface changes

6 smoothly. Figure 13 shows the shape of the sound insulator along with the sound field

7 and boundary conditions in the simulation. Since the sound field, vibrating surface,

8 and sound insulator were symmetric with respect to the $x z$-plane, only one side of the

9 symmetry plane was simulated so as to reduce the computational cost. Therefore, the

10 widths of the vibrating surface and sound insulator were $5 \mathrm{~mm}$, i.e., half the width

11 of the proposed acoustic transducer. The length of the vibrating surface was identical

12 to the length $L$ of the proposed acoustic transducer. The flow resistance $R_{\mathrm{f}}$ of the

13 sound insulator, assumed to be rock wool, was $1 \times 10^{6} \mathrm{~Pa} \cdot \mathrm{s} / \mathrm{m}^{2}$, and the characteristic

14 impedance and propagation constant of the porous material were calculated using Eqs.

15 (6)-(10). The maximum thickness of the sound insulator was $8.96 \mathrm{~mm}$, which was one-

16 quarter of the wavelength of the bending wave when $f=6 \mathrm{kHz}$. In addition, the slope

17 length of the sound insulator was $80 \mathrm{~mm}$. The boundary of the sound field was assumed

18 to satisfy the absorbing boundary condition. The vibrating surface was surrounded by

19 a rigid wall. The distribution of the particle velocity at a distance of $1 \mathrm{~mm}$ from the

20 sound insulator $(z=9.96 \mathrm{~mm})$ was calculated. 


\section{$1 \quad 5.2$ Results}

2 Figure 14 shows the distributions of the particle velocity at a distance of $1 \mathrm{~mm}$ from

3 the sound insulator $(z=9.96 \mathrm{~mm})$ when $f=6 \mathrm{kHz}$. Figures 14(a) and 14(b) show the

4 distributions of the particle velocity obtained without and with the sound insulator,

5 respectively. The particle velocity at the flat part was normalized as 1 . The solid line

6 and dashed line respectively show the ideal value obtained using the Rayleigh integral

7 in the previous section and the simulation result obtained by FEM simulation. The

8 chain line shown in Fig. 14(b) shows the result obtained by FEM simulation in the case

9 of inputting the optimized function in the vibrating surface. The chain line cannot be

10 seen because it is in exact agreement with the solid line obtained using the Rayleigh

11

12 integral. In addition, in the result obtained without the sound insulator, the distribution of the particle velocity at $z=9.96 \mathrm{~mm}$ was in good agreement with the ideal value in that the particle velocity increased at the edge of the vibrating plate, as shown in Fig. 14(a). The results suggest that it is appropriate to calculate the distribution of the particle velocity by FEM simulation. From Figs. 14(a) and 14(b), it can be seen that the distribution of the particle velocity was asymmetric regardless of the presence of the sound insulator because the phase of the vibrating surface was also asymmetric. In the result obtained with the sound insulator, the right part of the slope was in good agreement with the ideal value, as shown in Fig. 14(b). However, only the left part of 20 the slope increased rapidly despite the symmetry of the sound insulator. The increase 21 in particle velocity was presumably due to the interference of the leaking wave from the 22 edge of the vibrating surface and from the thin part of the sound insulator. According to these results, even if a symmetric sound insulator is placed in the evanescent wave 
1 field, it cannot produce a symmetric distribution of the particle velocity field.

2 Furthermore, the sound radiation from the vibrating surface with the distribution 3 of the particle velocity shown in Fig. 14(b) was obtained using the Rayleigh integral. 4 Figure 15 shows the distributions of the sound pressure level on the $x z$-plane when $f=$ $56 \mathrm{kHz}$. Figures 15(a) and 15(b) show the simulation results obtained with the optimized 6 window and with the sound insulator, respectively. The maximum sound pressure level 7 in each sound field was normalized as $0 \mathrm{~dB}$, and the sound pressure level was shown 8 down to $-50 \mathrm{~dB}$. It can be seen that the sound field obtained by the sound insulator 9 shown in Fig. 15(a) was in good agreement with that shown in Fig. 12(b). On the other hand, the suppression effect of the sound insulator seems to be small in comparison with

the optimized window function in this simulation as shown in Figs. 15(a) and 15(b). Nevertheless, the area where the sound pressure level decreased from -30 to $-50 \mathrm{~dB}$ was limited in comparison with the result obtained without the sound insulator shown in Fig. 12(a).

\subsection{Discussion}

According to the results, it was clarified that the sound insulator can control the distribution of the particle velocity via its thickness distribution. In addition, it was suggested that a sound insulator made of a porous material can suppress sound radiation by placing it above the vibrating plate. However, it is presumed that the amplitude of the evanescent sound field does not decrease monotonically with increasing thickness of the porous material when the material is placed in the evanescent sound field. Furthermore, the sound insulation characteristics vary intricately with the physical properties of the 
1 porous material, such as the density, the diameter of the fibers, and the percentage

2 volume of air pores. Therefore, the attenuation characteristics of the evanescent sound

3 field in the porous material should be analyzed in more detail. For this reason, it re-

4 mains a challenge to propose a theoretical method for calculating the shape of a sound

5 insulator that gives an identical distribution to the window function optimized by the

6 evaluation function. To further verify the applicability of the evaluation function for

7 calculating the optimized window function, we plan to propose a theoretical equation

8 for calculating the shape of the sound insulator using the optimized window function

9 while considering the physical properties and insulation characteristics of the sound

10 insulator as a future work.

11

\section{Conclusions}

In this study, a method of suppressing sound radiation to the far field of a near-field acoustic communication system using an evanescent sound field was proposed. A sound wave radiates over an evanescent sound field because of the broadening of the wavenumber spectrum caused by the presence of a discontinuity in the particle velocity. Therefore, in order to suppress sound radiation, we calculated an optimum window function giving the particle velocity field near a vibrating plate. We investigated a suitable method for calculating the sound pressure level at the far field, and the sound pressure level at the far field with respect to the frequency was calculated for different window shapes. A window function in which the maximum sound pressure level at the far field 21 has the smallest value in the frequency band $1 \leq f \leq 10 \mathrm{kHz}$ was obtained, and it was defined as the optimized window function. It was confirmed that the optimized window 
1 function can limit the area in which acoustic data is transmitted while securing high

2 sound pressure level near the vibrating plate. Additionally, it was found by simulation

3 using the three-dimensional FEM that sound radiation can be suppressed by placing a

4 sound insulator above the vibrating surface. As further study, we will theoretically in-

5 vestigate the method for calculating the shape of a sound insulator using the optimized

6 window function while considering the physical properties and insulation characteristics 7 of the sound insulator. 


\section{References}

1) G. Madlmayr, J. Langer, C. Kantner, and J. Scharinger, IEEE 3rd Int. Conf. Availability, Reliability and Security, 2008, p. 642.

2) J. Ondrus and Y. Pigneur, IEEE 6th Int. Conf. Management of Mobile Business, 2007, p. 43.

3) J. Cho, K. Min, and S. Kim, IEICE Trans. Commun. E89-B, 598 (2006).

4) A. Fujii, N. Wakatsuki, and K. Mizutani, Jpn. J. Appl. Phys. 53, 07 KB07 (2014).

5) A. Fujii, N. Wakatsuki, and K. Mizutani, Jpn. J. Appl. Phys. 54, 07 HB08 (2015).

6) D. H. Trivett, L. D. Luker, S. Petrie, A. L. Van Buren, and J. E. Blue, J. Acoust. Soc. Am. 87, 2535 (1990).

7) K. Nakamura and S. Hongo, Jpn. J. Appl. Phys. 37, 2853 (1998).

8) K. Yamada, D. Yamazaki, and K. Nakamura, Jpn. J. Appl. Phys. 43, 2996 (2004).

9) H. Matsuoka, Y. Nakashima, and T. Yoshimura, Advances in Multimedia Modeling (Springer, Heidelberg, 2008) p. 498.

10) N. Shinmen, T. Ebihara, and K. Mizutani, Proc. IEEE Global Conf. Consumer Electronics, 2012, p. 152.

11) A. Fujii, N. Wakatsuki, and K. Mizutani, Proc. 20th Int. Congr. Sound and Vibration, 2013, R25-344.

12) K. Koyama, T. Yamamoto, Y. Tamura, and Y. Wada, Jpn. J. Appl. Phys. 27, 218 (1988).

13) K. Saijyou, M. Egawa, and S. Yoshikawa, Jpn. J. Appl. Phys. 38, 3036 (1999).

14) M. Noguchi, K. Mizutani, K. Nagai, and Y. Yamashita, Jpn. J. Appl. Phys. 38, 
1

3

$3111(1999)$.

15) M. Noguchi, K. Mizutani, K. Nagai, and W. Cao, Jpn. J. Appl. Phys. 40, 3562 (2001).

16) T. Ohbuchi, T. Ebihara, K. Mizutani, N. Wakatsuki, and H. Masuyama, Jpn. J. Appl. Phys. 46, 4629 (2007).

17) T. Ohbuchi, T. Ebihara, K. Mizutani, N. Wakatsuki, and H. Masuyama, Jpn. J. Appl. Phys. 48, 07GC03 (2009).

18) T. Ohbuchi, T. Ebihara, K. Mizutani, N. Wakatsuki, and H. Masuyama, Jpn. J. Appl. Phys. 49, 07HC06 (2010).

19) S. Harigane, R. Miyasaka, S. Yoshizawa, and S. Umemura, Jpn. J. Appl. Phys. 52, $07 \mathrm{HF} 07$ (2013)

20) R. Miyasaka, J. Yasuda, M. Syahid, S. Yoshizawa, and S. Umemura, Jpn. J. Appl. Phys. 53, 07KF24 (2014).

21) G. R. Harris, J. Acoust. Soc. Am. 70, 10 (1981)

22) M. Yoshioka and T. Kikuchi, Jpn. J. Appl. Phys. 51, 07 GB03 (2012).

23) X. Guo, Y. Mizuno, and K. Nakamura, Jpn. J. Appl. Phys. 52, 127301 (2013).

24) J. W. Goodman, Introduction to Fourier Optics (McGraw-Hill, New York, 1996) p. 73 .

25) M. E. Delany and E. N. Bazley, Appl. Acoust. 3, 105 (1970).

26) Y. Miki, J. Acoust. Soc. Jpn. (E) 11, 1 (1990). 
1 Fig. 1. Near-field acoustic communication system using an evanescent sound field

2 generated near the surface of a vibrating plate.

3 Fig. 2. Frequency characteristics of the wavenumber $k_{\mathrm{p}}$ of a bending wave

4 propagating on an infinite acrylic plate with a thickness of $2 \mathrm{~mm}$.

5 Fig. 3. Sound generation from a bending wave propagating on a finite plate. (a) A 6 sound wave is radiated similarly to a beam with a uniform level and a constant angle 7 when the wavenumber of the bending wave $k_{\mathrm{p}}$ is smaller than that of the plane wave in 8 the sound field $k$. (b) An evanescent sound field is generated when $k_{\mathrm{p}}$ is larger than $k$.

9 Fig. 4. Wavenumber spectrum of bending wave propagating on an infinite vibrating

10 plate with wavenumber $k_{\mathrm{p}}$ and that obtained by applying a finite rectangular window to the particle velocity field.

Fig. 5. Window functions: triangular window, Hann window, cosine window, and Blackman window.

Fig. 6. Wavenumber spectra of window functions: (a) triangular window, (b) Hann window, (c) cosine window, and (d) Blackman window.

Fig. 7. Example of a window function with a shoulder part consisting of a cosine roll-off.

Fig. 8. Position of the vibrating plate of the proposed acoustic transducer on the $x z$-plane and the calculation area of the sound pressure level at the far field.

Fig. 9. Angular characteristics of sound pressure level at frequencies of (a) $f=6 \mathrm{kHz}$ 21 and (b) $f=30 \mathrm{kHz}$.

22 Fig. 10. Sound pressure level at the far field from the vibrating plate with respect to the frequency for the (a) triangular-type window, (b) cosine roll-off window, (c) 
1 cosine-type window, and (d) Blackman-type window.

2 Fig. 11. Maximum value $L_{\max }$ with respect to the edge length of the window in the

3 frequency band $1 \leq f \leq 10 \mathrm{kHz}$.

4 Fig. 12. Distributions of sound pressure level on the $x z$-plane $(y=0)$ (a) without

5 optimized window and (b) with optimized window.

6 Fig. 13. Shape of the sound insulator along with the sound field and boundary

7 conditions in the simulation using the three-dimensional FEM.

8 Fig. 14. Distributions of the particle velocity at a distance of $1 \mathrm{~mm}$ from the sound

9 insulator $(z=9.96 \mathrm{~mm}$ ) when $f=6 \mathrm{kHz}$ (a) without sound insulator and (b) with

10 sound insulator.

11 Fig. 15. Distributions of sound pressure level on the $x z$-plane when $f=6 \mathrm{kHz}$ (a)

12 with optimized window and (b) with sound insulator. 


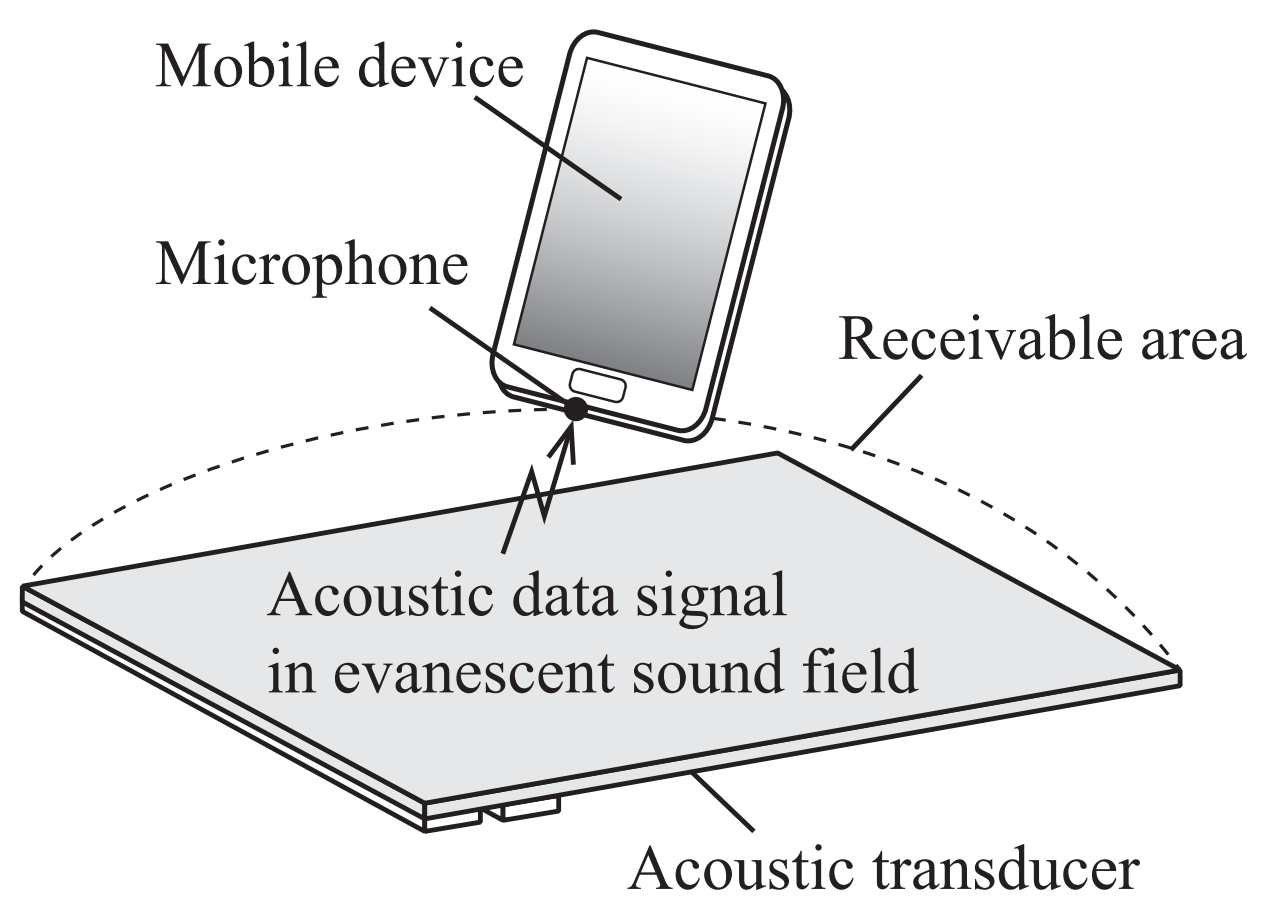

Fig. 1.

A. Fujii, N. Wakatsuki, and K. Mizutani 


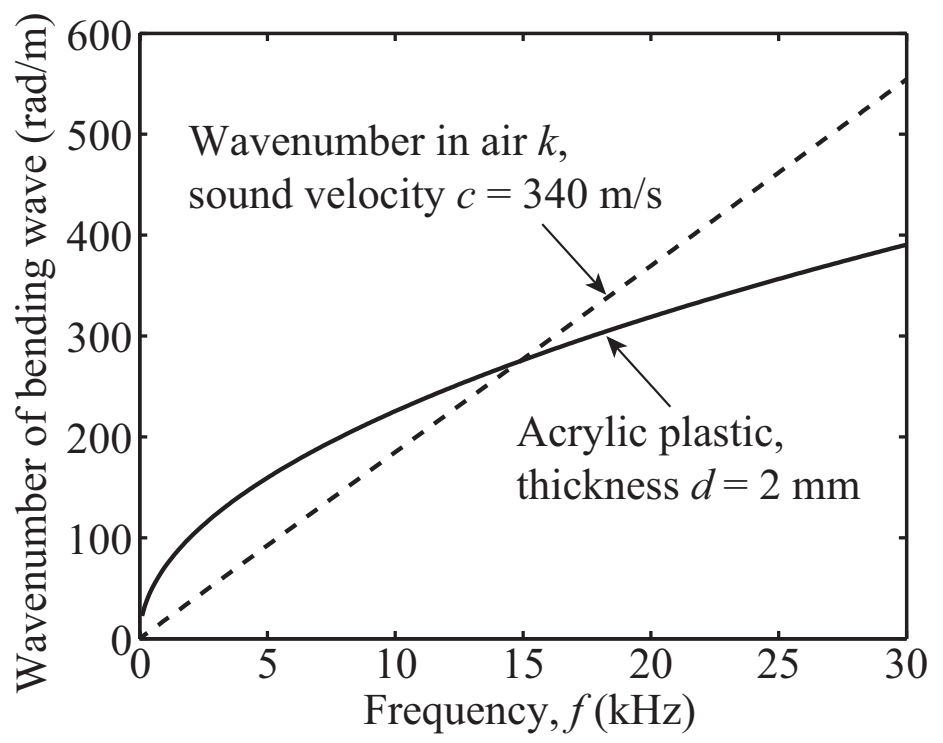

Fig. 2.

A. Fujii, N. Wakatsuki, and K. Mizutani 

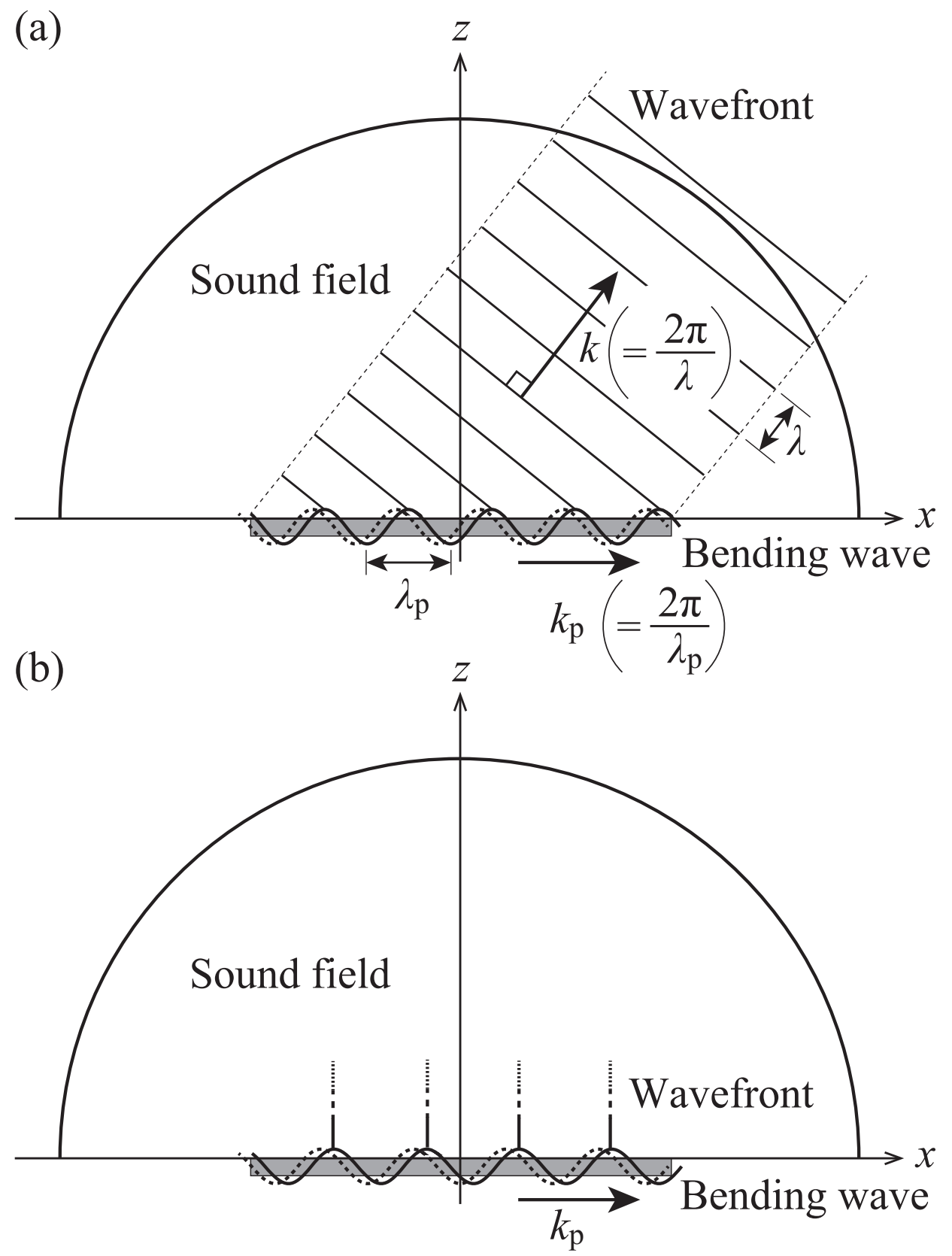

Fig. 3. 


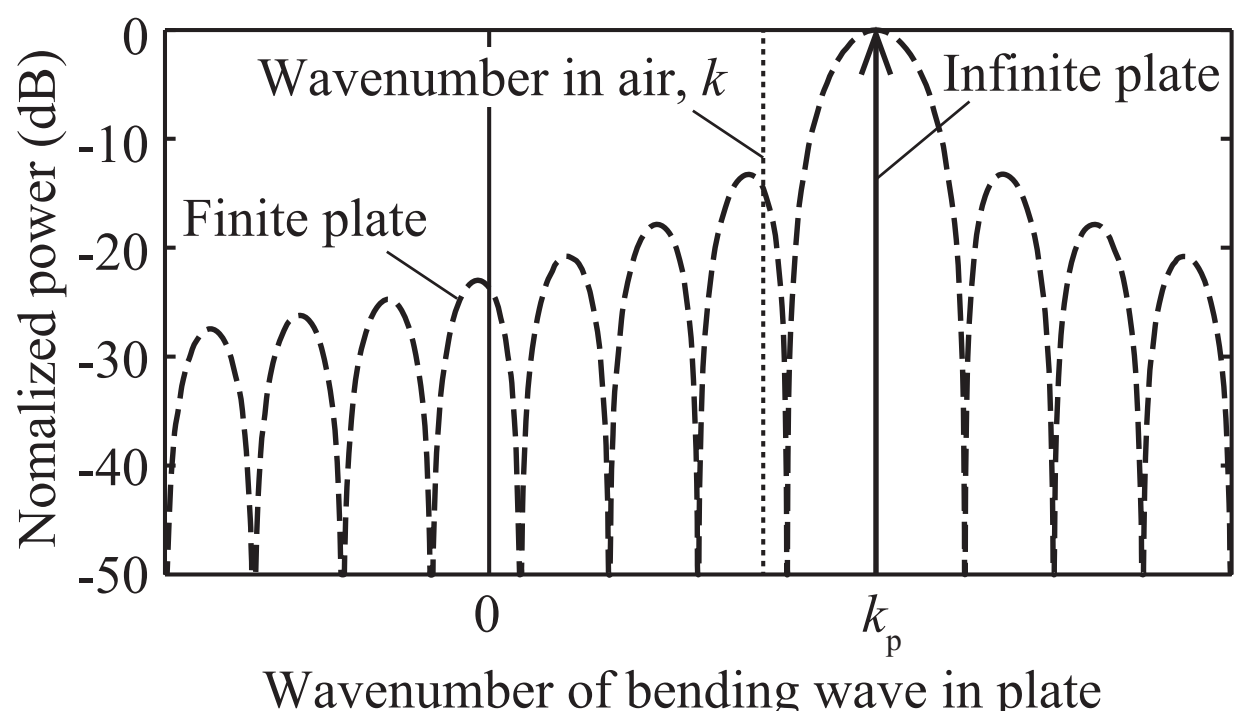

Fig. 4. 


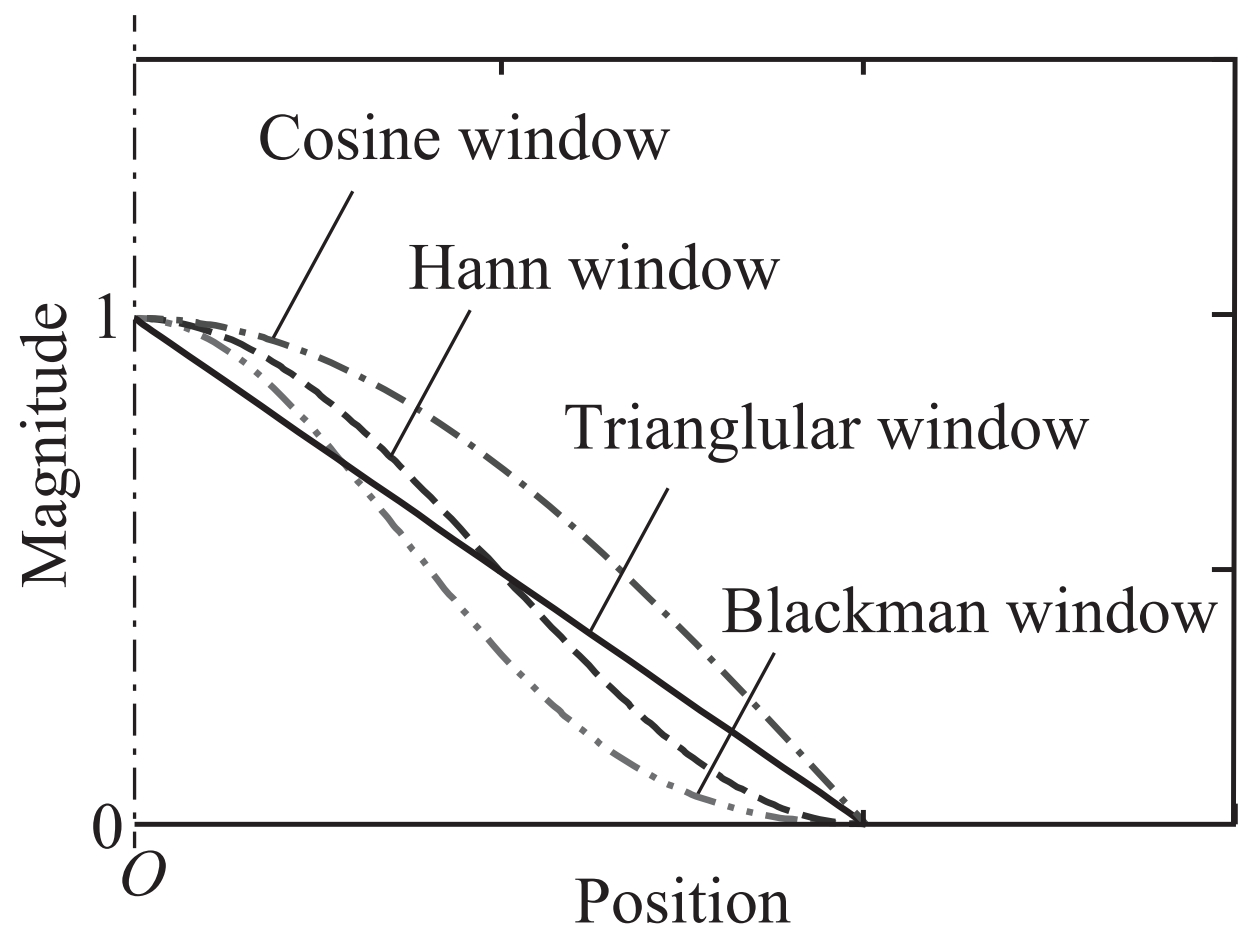

Fig. 5.

A. Fujii, N. Wakatsuki, and K. Mizutani 


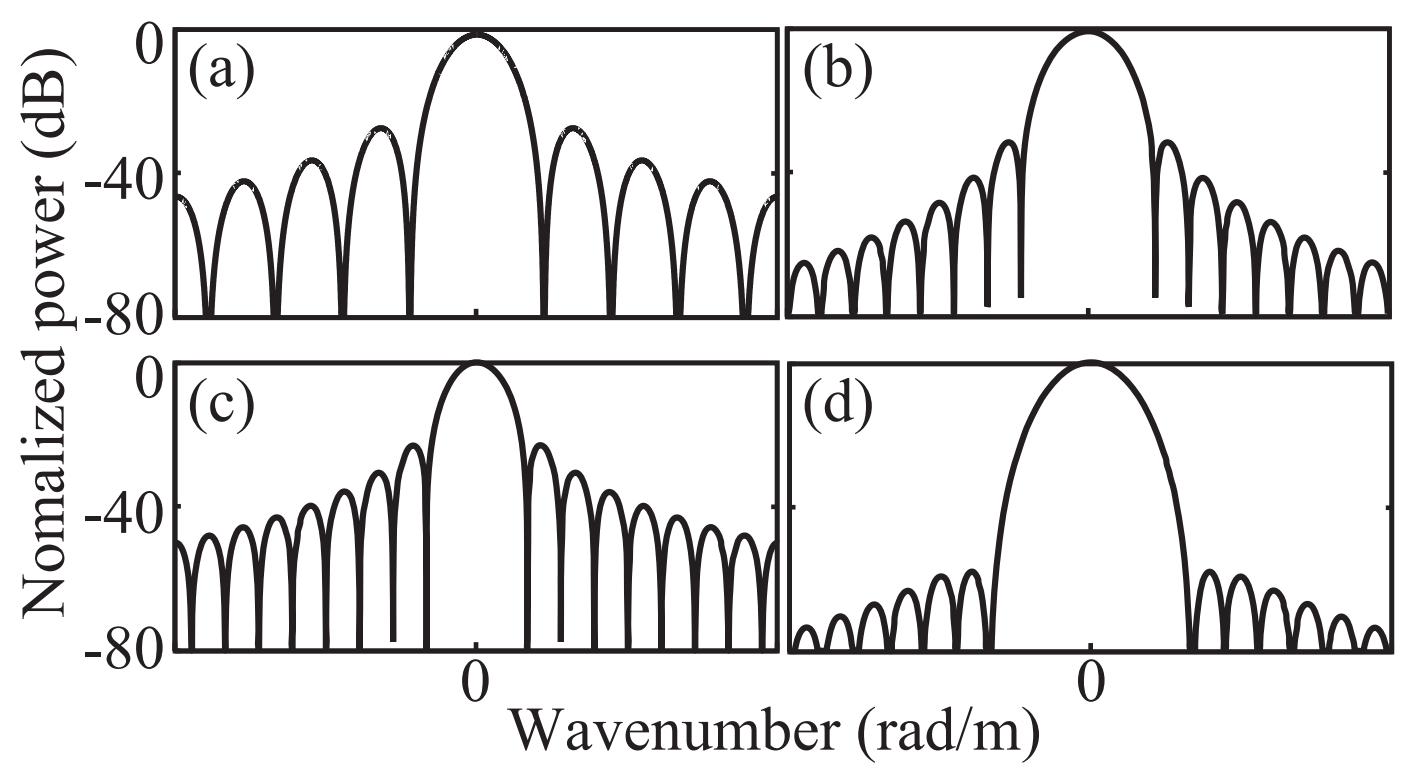

Fig. 6. 


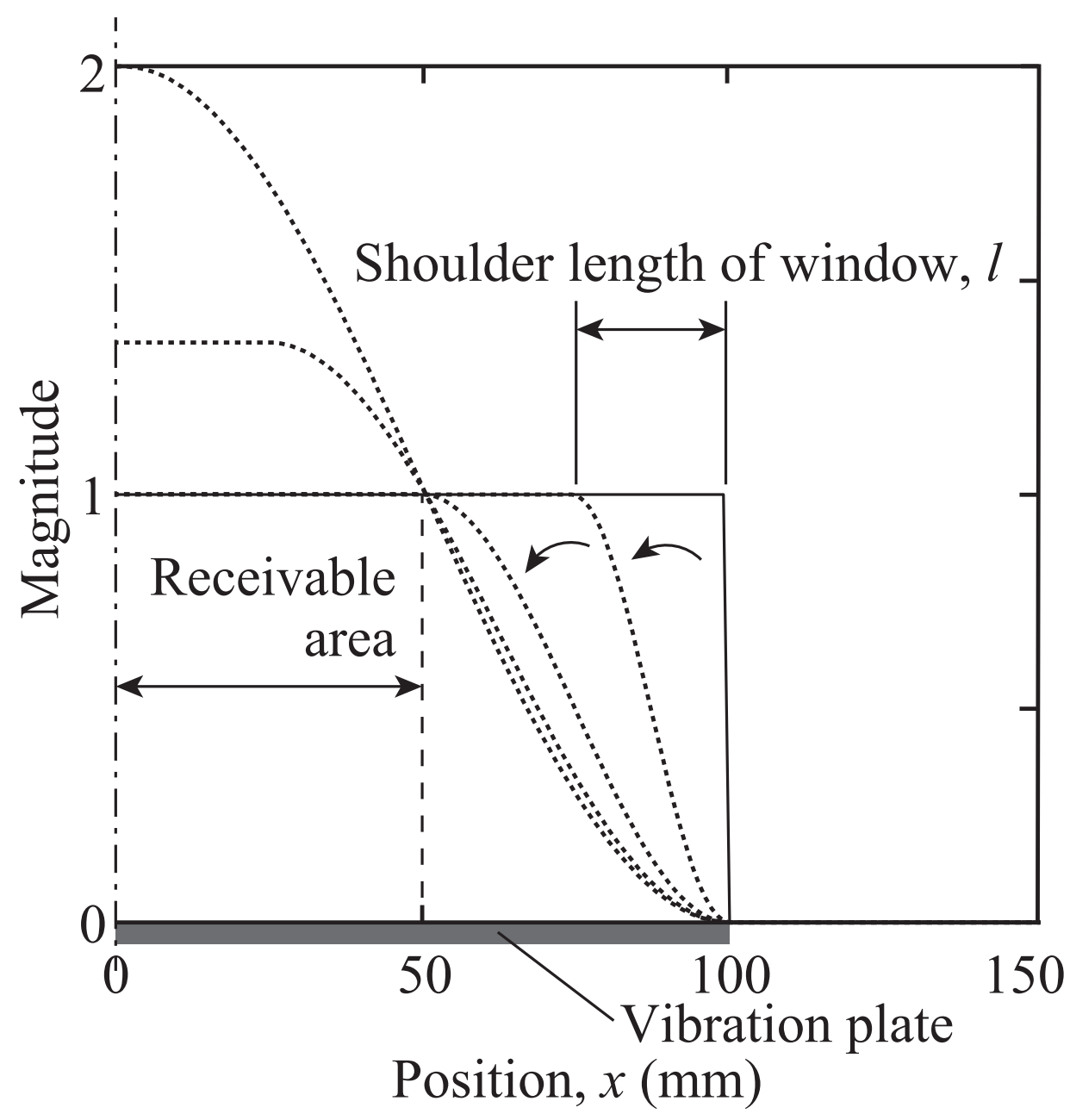

Fig. 7.

A. Fujii, N. Wakatsuki, and K. Mizutani 


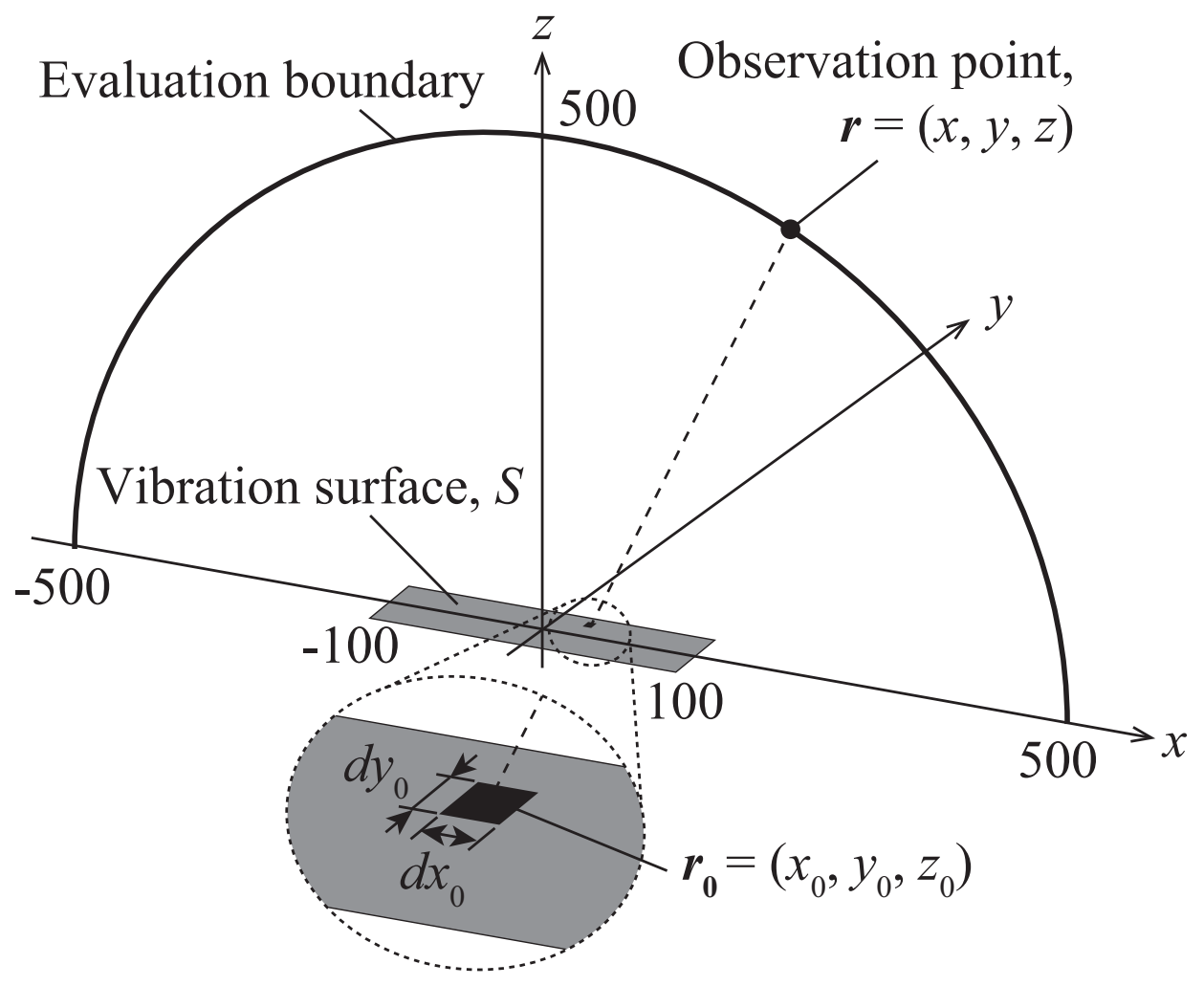

Fig. 8.

A. Fujii, N. Wakatsuki, and K. Mizutani 


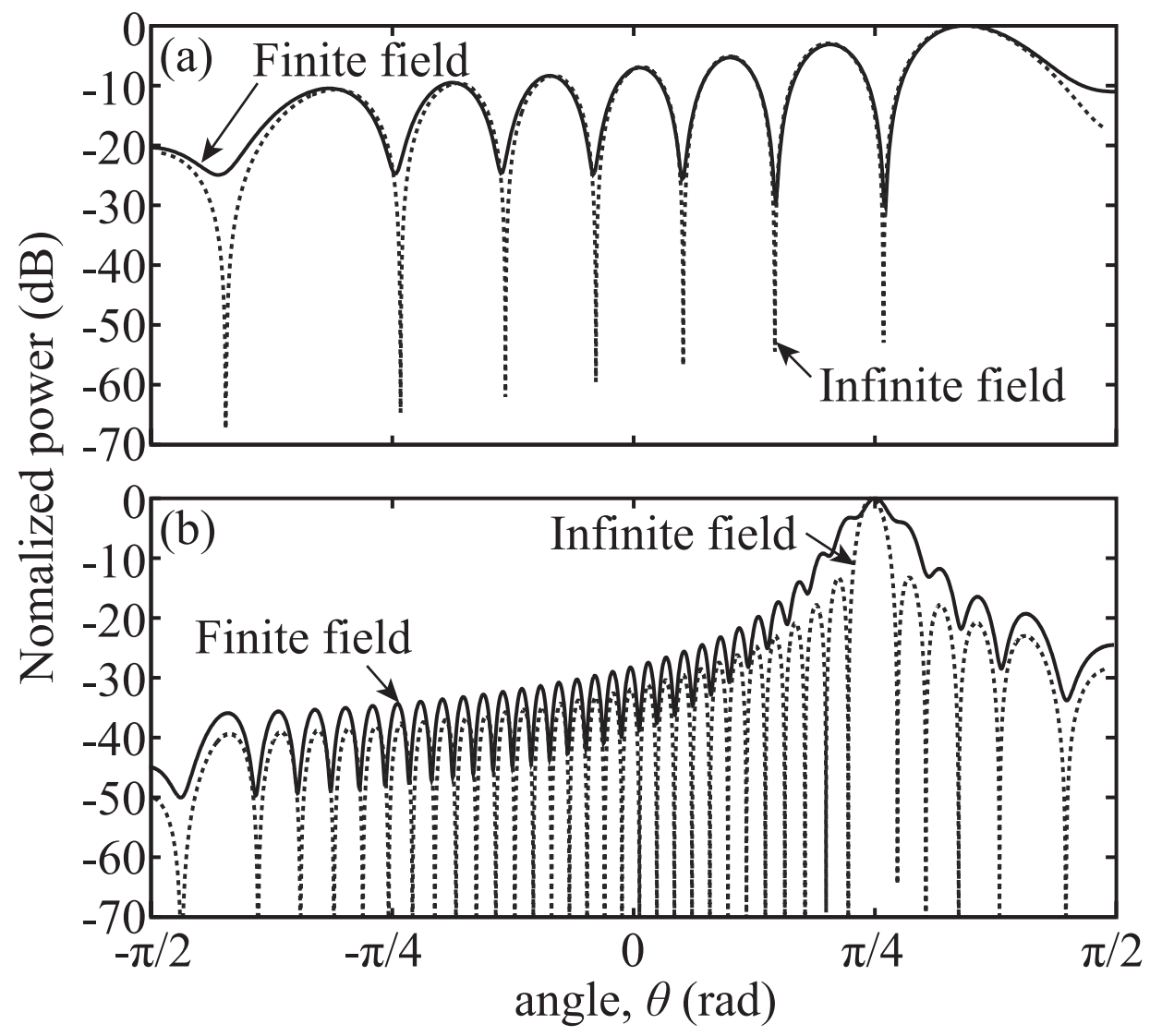

Fig. 9. 


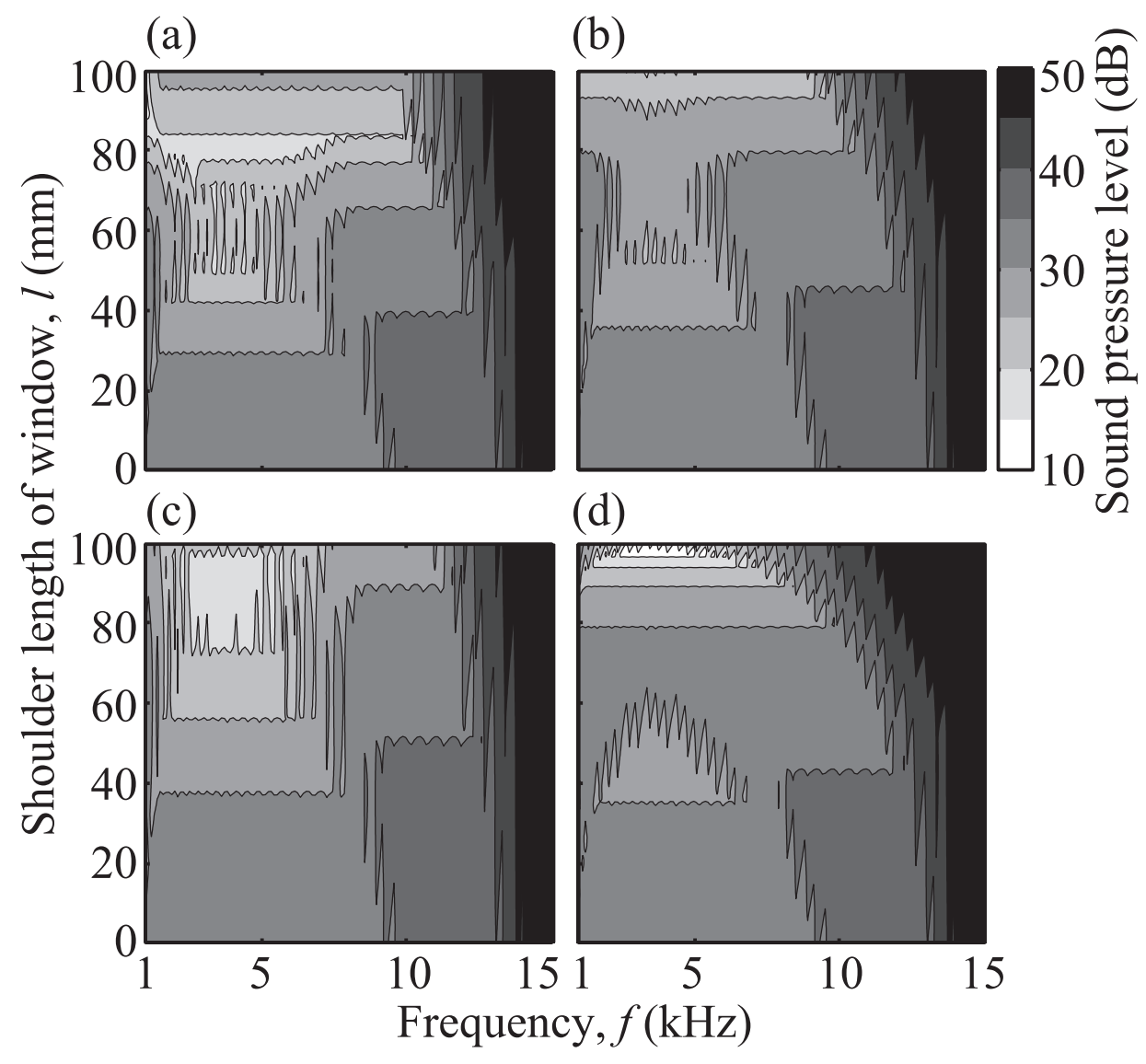

Fig. 10 .

A. Fujii, N. Wakatsuki, and K. Mizutani 


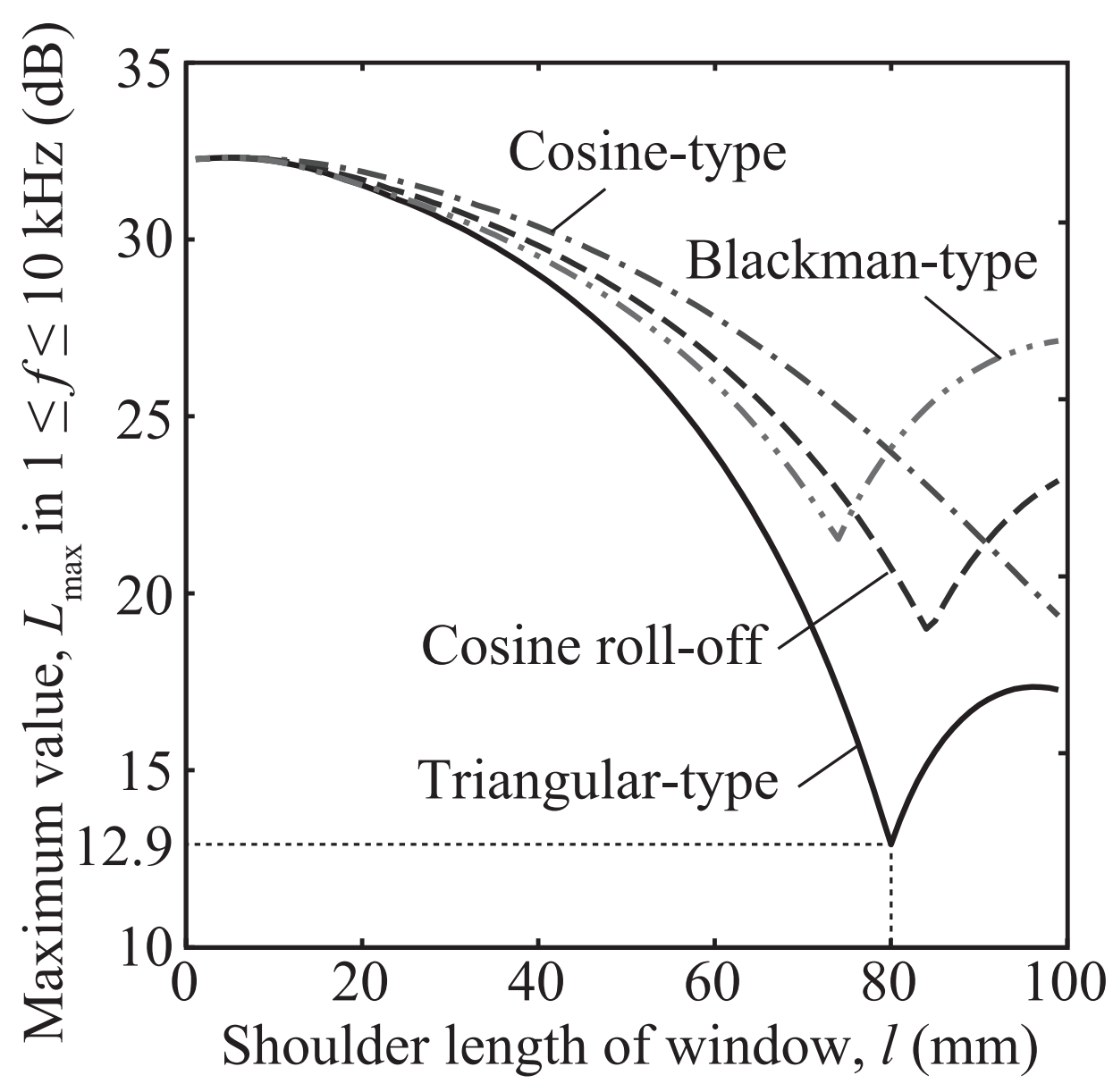

Fig. 11.

A. Fujii, N. Wakatsuki, and K. Mizutani 

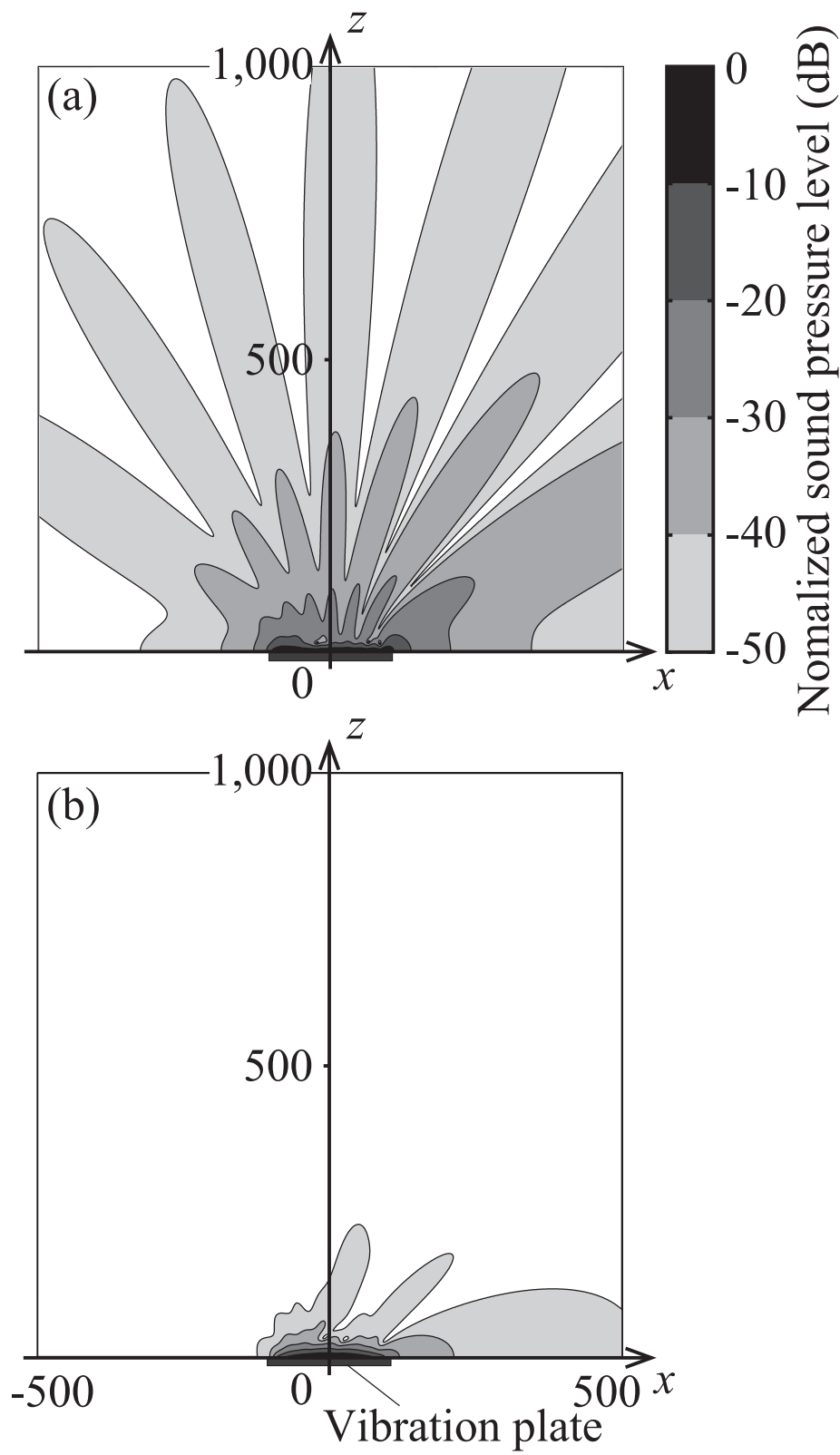

Fig. 12.

A. Fujii, N. Wakatsuki, and K. Mizutani 
Absorbing boundary (impedance of air)

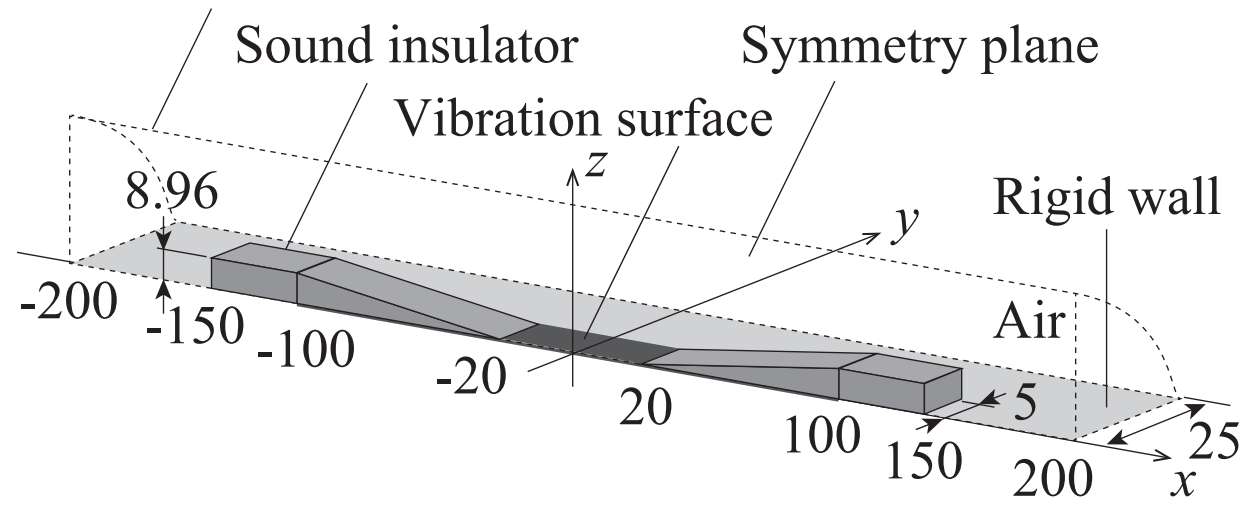

Fig. 13.

A. Fujii, N. Wakatsuki, and K. Mizutani 

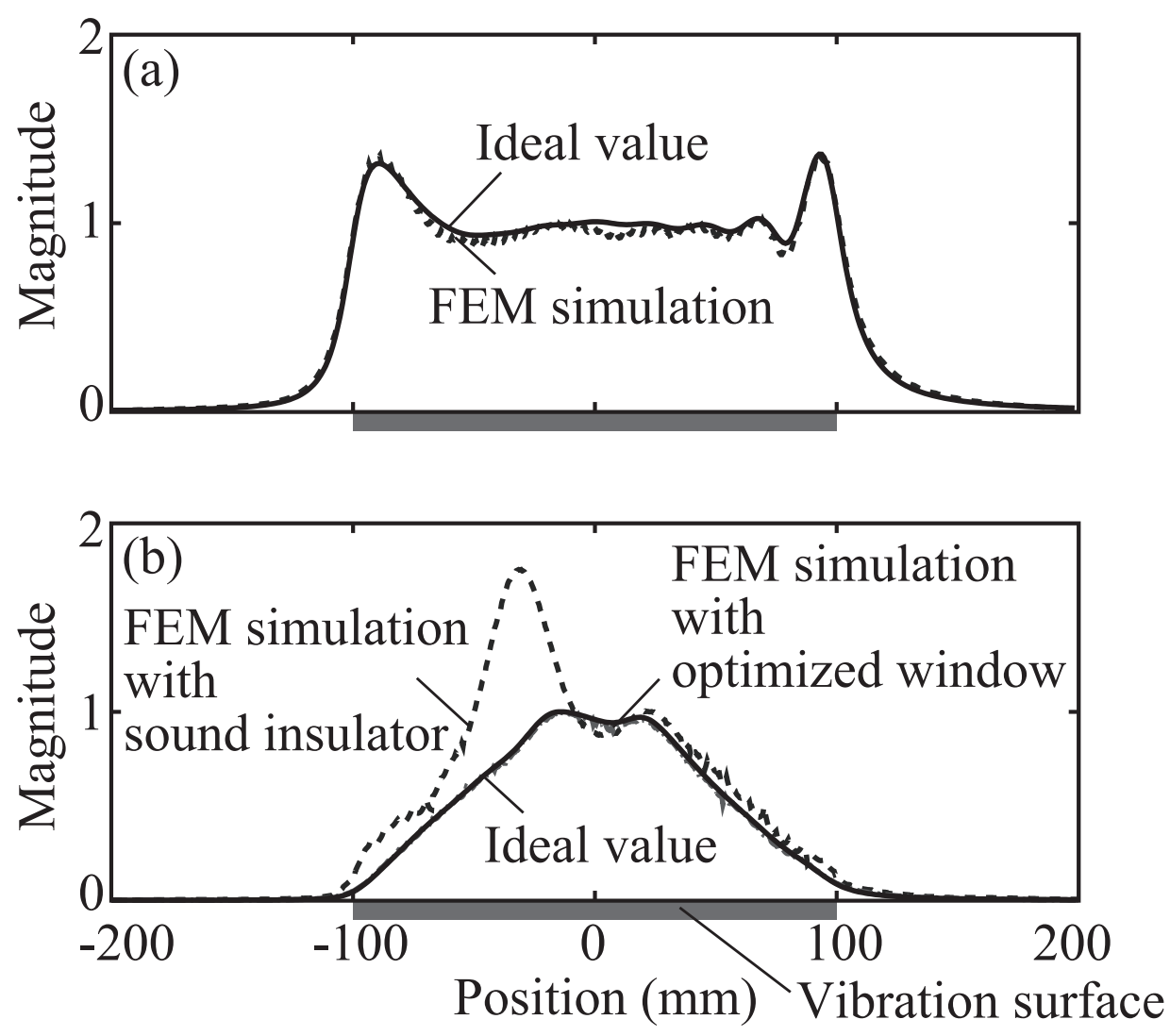

Fig. 14. 

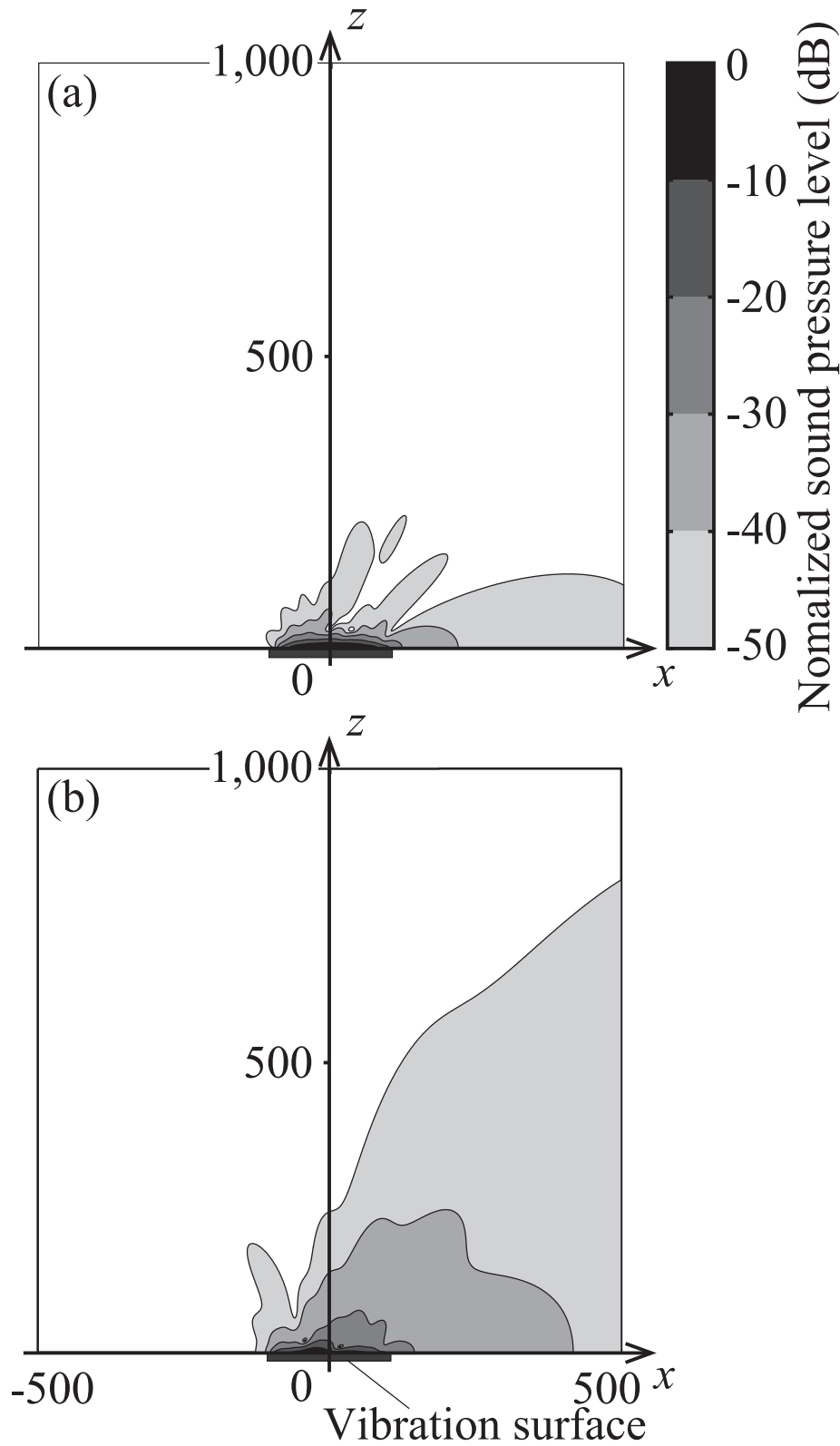

Fig. 15.

A. Fujii, N. Wakatsuki, and K. Mizutani 